\title{
Time to learn something new
}

D r. Patel's request to use desflurane, an accepted, albeit uncommon, inhalant anesthetic in dogs took Great Eastern's Attending Veterinarian (AV) and their IACUC by surprise; however it is reasonable to consider this request.

Desflurane is not labelled as an anesthetic for dogs, therefore its use in this species is considered extra-label and requires scientific justification. Patel's use of desflurane seems scientifically justified by his previous data as well as the desirable qualities he mentioned, but Great Easterns's IACUC should have the opportunity to evaluate this justification.

The AV and veterinary team have experience with isoflurane, which is an FDA approved anesthetic in dogs. Their concern about implementing a 'new to them' anesthetic for use in an extra-label manner has validity. There are, however, a number of publications describing the use of desflurane in dogs ${ }^{1,2}$; additionally, Patel and the veterinary team at his previous institution have experience as well as previous data with this drug. If Patel can demonstrate to Great Eastern's IACUC that desflurane is scientifically justified, the IACUC should approve its use and the veterinary team at
Great Eastern should avail themselves of the opportunity to learn from the team at. Patel's previous institution and gain experience with the use of desflurane in dogs.

The compromise proposed by the IACUC Chair is a good start. Training of the veterinary staff to gain experience with the new anesthetic is warranted for the safely of the animals, to reduce apprehension of the staff, and for the generation of consistent experimental data. The use of additional animals for training as proposed by the Chair is an option; however, as anesthesia, per se, is neither painful, nor distressful it also seems reasonable to gain anesthesia training on animals that will later be enrolled in Patel's study, thereby eliminating the need to purchase and use additional research animals.

With regard to funding for this training, the investigator and his team seem adequately trained and it is the veterinary staff that requires training. The Guide for the Care and Use of Laboratory Animals puts the responsibility for training on the Institution ${ }^{3}$. Generally this means that the Institution, and not the investigator, is responsible for the financial support required.
Going forward, a phone discussion with the previous institution's AV and/or veterinary team affords a reasonable way to begin learning about desflurane and how it compares to isoflurane in use and in monitoring depth of anesthesia. A discussion of monitoring equipment and how the animal's physiological parameters differ while under anesthesia with each agent should occur. This conversation may alleviate most if not all of the concerns around using desflurane and would be time well spent and perhaps money saved on additional training.

Karen L Moodie

Center for Comparative Medicine and Research, Dartmouth College, Lebanon, NH, USA.

e-mail:Karen.L.Moodie@dartmouth.edu

Published online: 25 February 2020

https://doi.org/10.1038/s41684-020-0487-3

\section{References}

1. Lopez, L. A., Hofmeister, E. H., Pavez, J. C. \& Brainard, B. M. Am J Vet Res. 70, 1339-44, https://doi.org/10.2460/ajvr.70.11.1339 (2009).

2. Hammond, R. A. et al. J. Vet. Anaesth. 21, 21-23 (1994).

3. Institute for Laboratory Animal Research. Guide for the Care and Use of Laboratory Animals. 8th edition (National Academies Press, Washington, D.C., USA, 2011). 